\title{
Antibiotic-resistant opportunistic bacteria in the coastal zone of Lake Baikal
}

LIMNOLOGY FRESHWATER

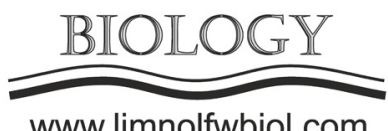

\author{
Shtykova Yu.R., Suslova M.Yu., Podlesnaya G.V., Krasnopeev A.Yu., Belykh O.I. \\ Limnological Institute, Siberian Branch of the Russian Academy of Sciences, Ulan-Batorskaya Str., 3, Irkutsk, 664033, Russia
}

\begin{abstract}
This work presents the diversity and antibiotic sensitivity of opportunistic bacteria isolated from plankton and biofilms in the coastal zone of Lake Baikal. Using modern identification methods (molecular genetic and mass spectrometry analyses), we determined that bacteria identified in biotopes belong to the genera Escherichia, Citrobacter, Hafnia, Enterobacter, Shigella, Yersinia, Serratia, Pseudomonas, Acinetobacter, Bacillus, Enterococcus, Staphylococcus, which are mostly the cause of community-acquired and nosocomial infections. The bulk of isolates showed polyresistance to widespectrum antibiotics ( $\beta$-lactams and sulphanilamides).
\end{abstract}

Keywords: opportunistic bacteria, antibiotic resistance, water pollution, Lake Baikal.

\section{Introduction}

The spread of antibiotic-resistant bacteria (ARB) in the environment is one of the most important and serious problems in the fields of medicine and public health (Pazda et al., 2019). Wastewater is the main source of microbial pollution of environmental objects. Despite the disinfection at the final stage of the wastewater treatment, terminal effluents bring to the environment a large number of bacteria having antibiotic resistance genes and contributing to the spread of these genes in environmental microbiomes (Woegerbauer et al., 2020). This leads to a decrease in the effectiveness of the treatment of bacterial infections and, consequently, an increase in morbidity and mortality (Pazda et al., 2019). An alarming trend of the ARB spread was also previously observed in the water area of Lake Baikal (Panasyuk et al., 2002; Parfenova et al., 2008; Verkhozina et al., 2014).

\section{Material and methods}

To form a collection of opportunistic bacteria, water and biofilms from different substrates were sampled from May to November 2018 in the coastal zone of Lake Baikal. Sampling stations were located mainly near the tourist objects and settlements. The isolates were identified using a set of methods, including molecular genetic and mass spectrometry analyses. To determine the antibiotic sensitivity of the strains, broad-spectrum antibiotics were used: $\beta$-lactams, azalides, tricyclic glycopeptides, aminoglycosides, chloramphenicols, sulphanilamides, tetracyclines, and fluoroquinolones. The study was carried out using a disk diffusion test specified in the MUK 4.2.1890-04 methodology guidelines.

\section{Results}

We obtained 94 bacterial strains from plankton and biofilms. Among them, we identified 18 genera of clinical significance. The bulk of the collection (51\%) consisted of gram-negative facultatively anaerobic gammaproteobacteria, order Enterobacterales (24\%) and genus Aeromonas (27\%). The genera Escherichia (E. coli), Citrobacter (C. braakii), Hafnia (H. alvei), Enterobacter, Shigella, Yersinia, Serratia, Lelliottia, and Leclercia represented the diversity of enterobacteria. Gram-positive aerobic and facultative anaerobic firmicutesof thegeneraBacillus, Enterococcus(E.faecium), Aerococcus, Staphylococcus, and Exiguobacterium were less common (32\%). A smaller proportion of the strains $(17 \%)$ were gram-negative non-fermenting gammaproteobacteria (the genera Pseudomonas ( $P$. aeruginosa), Acinetobacter and Stenotrophomonas). Most strains of gram-negative bacteria showed polyresistance to penicillins (85\% to benzylpenicillin, $61 \%$ to ampicillin and $57 \%$ to amoxicillin). Among gramnegative non-fermenting bacteria, $93 \%$ of the strains were resistant to benzylpenicillin. More than half of gram-positive strains were resistant to cephalosporins ( $49 \%$ to cefazolin and $51 \%$ to cefotaxime). Notably, the cultures of the genus Enterococcus showed the highest resistance to cephalosporins ( $87 \%$ to cefazolin and $80 \%$ to cefotaxime). A larger proportion of all studied strains showed resistance to sulphanilamide (gram-

*Corresponding author.

E-mail address: adress@email.com (F.L. Author) 
negative bacteria $-67 \%$, gram-positive bacteria - 55\%). Moreover, all enterobacteria cultures were resistant to this antibiotic.

\section{Discussion and conclusions}

ARB detected in biotopes of Lake Baikal are an etiological factor of opportunistic infections, in the treatment of which penicillins and cephalosporins are often used. This is extremely important that the detected ARB can carry antibiotic resistance genes. Discharge of wastewater into Lake Baikal, which contains ARB even after the treatment, leads to the spread of these genes in the ecosystem, as evidenced by the regular detection of ARB in the water area of the lake (Panasyuk et al., 2002; Parfenova et al., 2008; Verkhozina et al., 2014). Lake Baikal is the largest source of drinking water; therefore, the standard approaches to disinfecting the effluents discharged into it are insufficient. To combat the spread of ARB in Lake Baikal, it is necessary to study the processes of resistance gene transfer in its ecosystem and develop biocontrol technologies in wastewater treatment systems.

\section{Acknowledgments}

The study was carried out within the framework of State Task No. 0345-2019-0003 (AAAA-A16-116122110061-6). This work was supported by RFBR grant no. 16-54-44035_mong_a.

\section{References}

Panasyuk E.Yu., Drucker V.V., Parfenova V.V. et al. 2002. Biodiversity and distribution of bacteria of Enterobacteriaceae family and of non-enzymatic group in Lake Baikal. Sibirskiy Ekologicheskiy Zhurnal [Siberian Journal of Ecology] 4: 485-490. (in Russian)

Parfenova V.V., Kravchenko O.S., Pavlova O.N. 2008. Distribution and antibiotic resistance of Enterococcus isolated from Lake Baikal. Sibirskij Medicinskij Zhurnal [Siberian Medical Journal] 3: 78-81. (in Russian)

Pazda M., Kumirska J., Stepnowski P. et al. 2019. Antibiotic resistance genes identified in wastewater treatment plant systems - a review. Science of the Total Environment 697. DOI: 10.1016/j.scitotenv.2019.134023 M

Verkhozina E.V., Verkhozina V.A., Savilov E.D. et al. 2014. Antibiotic resistance of microbial community of the Lake Baikal ecosystem in the area of Listvyanka, Slyudyanka and Baikalsk. Acta Biomedica Scientifica 3: 62-65. (in Russian)

Woegerbauer M., Bellanger X., Merlin C. 2020. CellFree DNA: an underestimated source of antibiotic resistance gene dissemination at the interface between human activities and downstream environments in the context of wastewater reuse. Frontiers in Microbiology 11. DOI: 10.3389/ fmicb.2020.00671 